\title{
The role of temperature in plasmon sensors in physical and biological research
}

Received: 07 December, 2020

Accepted: 11 January, 2021

Published: 12 January, 2021

*Corresponding author: AD Suprun, Faculty of Physics, Taras Shevchenko National University of Kyiv, 64/13, Volodymyrska Street, Kyiv 01601, Ukraine, E-mail: saddas.new@gmail.com

https://www.peertechz.com

\section{Check for updates}

\section{Shmeleva and AD Suprun*}

Faculty of Physics, Taras Shevchenko National University of Kyiv, 64/13, Volodymyrska Street, Kyiv 01601, Ukraine

\section{Introduction}

Recently, sensors that use the phenomenon of plasmon resonance have been widely used [1]. In this case, biosensors are of particular interest [2].

The plasmon resonance method is attractive in that it has a sufficiently high sensitivity to changes in concentration (the most widespread use) of the medium under study (analyte). But this method can be no less attractive for research other properties. For example, the effects of temperature during laser irradiation. In such sensors, on the one hand, the temperature can significantly affect the frequency position of the plasmon resonance, which cannot be ignored. On the other hand, the "temperature effect" itself can be the basis for measuring physical quantities that are sensitive to temperature.

It is the effect of temperature on plasmon sensors, including biological ones, which will be discussed in this short review.

\section{Plasmon resonance}

The "classical" sensor circuit is considering (Figure 1).

The radiation enters through a glass prism (in the figure above) onto the surface of a metal layer (most often gold) up to $40 \mathrm{~nm}$ thick.

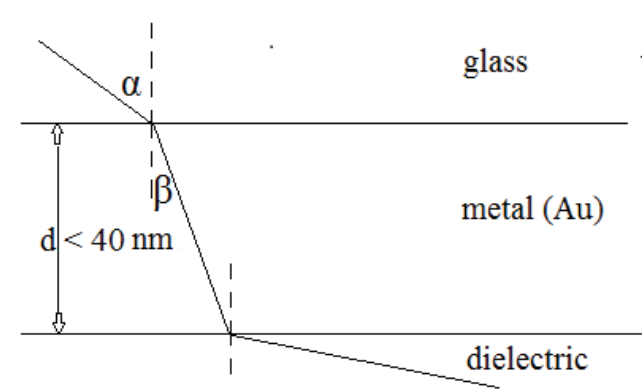

Figure 1: The simplest "optical" sensor circuit.
After passing through the metal layer, at a certain angle of incidence $\alpha$, a plasmon wave is excited on the lower surface (at the metaldielectric interface), which absorbs the input wave, and the intensity of the reflected beam from the interface metal-dielectric decreases significantly. Such a decrease is a sensory effect, since the plasmon frequency depends significantly on the dielectric constant of the dielectric [3]:

$$
\omega_{p r}=\omega_{p} / \sqrt{1+\varepsilon}
$$

Here $\omega_{p r}$ is the plasmon resonance frequency, $\omega_{p}=e \sqrt{4 \pi n_{e} / m_{e}}$ is the plasma frequency of the electron subsystem of the metal ( $e$ is the electron charge, $m_{\mathrm{e}}$ is its mass in the metal, $n_{\mathrm{e}}$ is the bulk density of electrons), $\varepsilon$ is the dielectric constant of the dielectric. As can be seen from formula (1), a change in $\varepsilon$ causes a change in $\omega_{p r}$, which causes a change in the angle at which the resonance occurs. That is why (due to the change in angle) this method is quite sensitive.

\section{Influence of temperature}

Since the penetration depth of radiation for gold exceeds $400 \mathrm{~nm}$ [4], a layer up to $40 \mathrm{~nm}$ thickness will, on the one hand, be sufficiently transparent for electromagnetic radiation, and, on the other hand, it is known [5] that absorption will be quite noticeable in the near-surface layer of such thickness and will cause an increase a temperature in it according to equality:

$$
T=T_{0}+\frac{2 \mathrm{q} \sqrt{\tau}}{\sqrt{\pi \rho \mathrm{C} \lambda_{\mathrm{c}}}} \exp (-k \mathrm{z})
$$

Here $\mathrm{q}$ is the radiation flux, $\tau$ is the pulse duration (or exposure time), $\rho$ is the bulk density of the material, $C$ is its specific heat capacity, $\lambda_{\mathrm{c}}$ is the thermal conductivity coefficient, $k$ is the absorption index (dimension $1 / \mathrm{m}$ ) associated with the dimensionless absorption coefficient $k$ by the ratio $k=\omega \kappa / c \equiv 2 \pi \kappa / \lambda \quad(\lambda$ is radiation 
wave-length), $z$ is the depth of penetration to the metal layer of the electromagnetic flux.

Using relation (2), it is possible to estimate that at minimum fluxes of laser radiation $q \sim 10^{11} \mathrm{~W} / \mathrm{m}^{2}\left(10^{7} \mathrm{~W} / \mathrm{cm}^{2}\right)$ and at pulse duration $\tau=143 \mathrm{fs}\left(143 \cdot 10^{-15} \mathrm{~s}\right)$, the near-surface layer of gold will be heated by $2^{\circ}$. But the applied fluxes of radiation and pulse durations can be much larger. So the heating of the gold layer can reach $60^{\circ}$. Due to the processes of heat transfer, the near-surface layer of the dielectric will also warm up to the same temperature.

This, in any case, cannot be ignored if "sensitivity" $€$ to temperature is significant.

\section{Dependence of the dielectric constant of conventional dielectrics on temperature}

For conventional (traditional) isotropic dielectrics Clausius and Massotti independently established the relationship:

$$
\frac{\varepsilon-1}{\varepsilon+2}=\frac{4 \pi}{3} n \alpha \text {. }
$$

Here $\varepsilon$ is the static dielectric constant; $n$ is the bulk density of molecules, that are polarized, $\alpha$ is the polarizability of an individual molecule.

In relation (3), the bulk density $n$ molecules should depend on temperature. This is quite obvious from the following considerations. With increasing temperature, thermal expansion of the material occurs. That is, the effective volume increases by one molecule. But the bulk density $n$ of molecules is reciprocal to this volume. That is, it decreases with increasing temperature.

By direct differentiation of relation (3) with respect to temperature, after some transformations using the relation (3) itself, one can obtain the differential equation: $\frac{d \varepsilon}{d T}=-\frac{\beta}{3}(\varepsilon-1)(\varepsilon+2)$. In this equation $\beta \equiv-\frac{1}{n} \frac{d n}{d T}$ is the coefficient of volume expansion, which in a wide temperature range (up to the melting point) also depends on temperature, but to increase the sensitivity of the method, the dependence $\beta$ on $T$ should be taken into account for all temperatures. The resulting equation unambiguously demonstrates a decrease in dielectric constant with temperature, since its right side is negative. From formula (1) it follows that in this case the plasmon frequency increases.

\section{Dependence of the dielectric constant of proteins and other organic dielectrics on the temperature}

In the case when the dielectric layer (in Figure 1 - its lower part) is formed by protein molecules, one speaks about biosensors [6,7]. Then, instead of relation (3), one should use the Langevin-Debye relation:

$$
\frac{\varepsilon-1}{\varepsilon+2}=\frac{4 \pi}{3} n \cdot\left(\alpha+\frac{p^{2}}{3 k_{B} T}\right)
$$

Here $P$ is the stationary dipole moment of an individual molecule, $K_{\mathrm{B}}$ is the Boltzmann constant.
This relationship, in contrast to (3), refers to polar dielectrics, that is, having a nonzero dipole moment in the absence of an external electric field. Proteins in this sense are highly polar dielectrics. In proteins there are so-called peptide groups with a stationary dipole moment $\sim 3 \mathrm{D}$ [8]. Symbol D signify for the unit of the dipole moment: $1 \mathrm{D}=1 \mathrm{e} \cdot \AA=$ $1.6 \cdot 10^{-29} \mathrm{C} \cdot \mathrm{m}$. This unit of dipole moment is called Debye. Since there are hundreds of such groups in a protein, its dipole moment can be tens or even hundreds of Debye, depending on the configuration of the protein molecule.

Relation (4) is used to determine the dielectric constant also in the case of other organic compounds, if they have a stationary dipole moment.

A feature of biosensors is that the bulk density of molecules $n$ may not change with temperature, or change very weakly, since each molecule can have a fixed position on the metal surface. In the case when the polarizability $\alpha$ of the molecule and its dipole moment $P$ do not depend on temperature, the differential equation for determining the dependence of $\varepsilon$ on $T$ will have the form: $\frac{d \varepsilon}{d T}=-\frac{4 \pi n p^{2}}{27 k_{B} T^{2}}(\varepsilon+2)^{2}$ . It also demonstrates a decrease in dielectric constant with increasing temperature, that is, an increase the plasmon resonance frequency.

\section{References}

1. Homola J, Yee SS, Gauglitz G (1999) Surface plasmon resonance sensors: review. Sensors and actuators B: Chemical. 54: 3-15. Link: https://bit.ly/3nzSbzl

2. Buzavaite-Verteliene $\mathrm{E}$, Plikusiene $\mathrm{I}$, Tolenis $\mathrm{T}$, Valavicius $\mathrm{A}$, Anulyte $\mathrm{J}$, et al (2020) Hybrid Tamm-surface plasmon polariton mode for highly sensitive detection of protein interactions. Optics Express 28: 29033-29043. Link: https://bit.ly/3nzH3Tu

3. Raether H (2013) Surface plasmons on smooth and rough surfaces and on gratings. Springer-Verlag Berlin An 140.

4. Ushakov NM, Vasilkov MY, Fedorov FS (2017) The influence of a thin gold film on the optical spectral characteristics of a porous anodic aluminum-oxide membrane. Technical Physics Letters 43: 648-651. Link: https://bit.ly/3q7K9zR

5. Suprun AD, Shmeleva LV, Razumova MA (2011) The influence of bulk absorption of substance on the threshold of destruction by the intensive pulse of electromagnetic radiation. Functional materials 18: 237-243. Link: https://bit.ly/3sbRMqX

6. Chen YT, Liao YY, Chen CC, Hsiao HH, Huang JJ (2019) Surface plasmons coupled two-dimensional photonic crystal biosensors for Epstein-Barr virus protein detection. Sensors Actuators, B Chem Link: https://bit.ly/3qeIPvj

7. Hong $\mathrm{CH}$, Tang MR, Hsu SH, Yang CH, Tseng CS, et al. (2019) Enhanced early immune response of leptospiral outer membrane protein LipL32 stimulated by narrow band mid-infrared exposure. Journal of Photochemistry and Photobiology B: Biology 198: 111560. Link: https://bit.ly/38zoURM

8. Davydov AS (1982) Biology \& quantum mechanics. Pergamon Press, Oxford, Uk, 229. Link: http://bit.ly/3idsog1

Copyright: @ 2021 Shmeleva LV, et al. This is an open-access article distributed under the terms of the Creative Commons Attribution License, which permits unrestricted use, distribution, and reproduction in any medium, provided the original author and source are credited. 